This item is the archived peer-reviewed author-version of:

\title{
A multilinear mixing model for nonlinear spectral unmixing
}

\section{Reference:}

Heylen Rob, Scheunders Paul.- A multilinear mixing model for nonlinear spectral unmixing IEEE transactions on geoscience and remote sensing / Institute of Electrical and Electronics Engineers [New York, N.Y.] - ISSN 0196-2892 - 54:1(2016), p. 240-251

Full text (Publishers DOI): http://dx.doi.org/doi:10.1109/TGRS.2015.2453915

Handle/Permalink: http://hdl.handle.net/10067/1303520151162165141 


\title{
A multilinear mixing model for nonlinear spectral unmixing
}

\author{
Rob Heylen, Paul Scheunders
}

\begin{abstract}
In hyperspectral unmixing, bilinear and linearquadratic models have become popular recently, and also the polynomial post-nonlinear model shows promising results. These models do not consider endmember interactions involving more than two endmembers, although such interactions might compose a nontrivial part of the observed spectrum in scenarios involving bright materials and complex geometrical structures such as vegetation and intimate mixtures. In this work, we present an extension of these models to include an infinite number of interactions. Several technical problems such as divergence of the resulting series can be avoided by introducing an optical interaction probability, which becomes the only free parameter of the model in addition to the abundances. We present an unmixing strategy based on this multilinear mixing model, present comparisons with the bilinear models and the Hapke model for intimate mixing, and show that in several scenarios, the multilinear mixing model obtains superior results.
\end{abstract}

\section{INTRODUCTION}

In hyperspectral unmixing applications, the aim is to decompose the spectrum observed in a pixel of a hyperspectral image into different contributions from elemental materials. Typically, one assumes that there exist a number of pure materials, named endmembers, and that each endmember comprises a certain fractional area of the instantaneous field of view (IFOV) of the pixel, known as the abundance of the endmember. The observed spectrum then depends of the spectra of the individual endmembers and their relative abundances in the pixel according to some mixing model.

The simplest mixing model assumes that there are no interactions between endmembers, and that each incoming light ray interacts with only a single material before reaching the observer. This leads to a linear mixing model (LMM), where the observed spectrum is a convex linear combination of the endmember spectra. Convexity arises due to two physical constraints: Since the abundances are relative areal fractions, they have to be non-negative, yielding the abundance nonnegativity constraint (ANC). Furthermore, one typically assumes an abundance sum-to-one constraint (ASC) as well, reasoning that the entire spectral signal has to be decomposed into endmember contributions, disallowing unknown signal components or scaling factors. Several authors consider this latter constraint too restrictive, and alleviate it by introducing a zero or shadow endmember, or by simply discarding this constraint. In all cases, the resulting set of pixel values allowed by the model will still be a convex set.

Due to its simplicity and intuitive interpretation, the LMM has become a very popular model for unmixing, and has shown great results in many scenarios, especially those involving large flat areas with clearly separated regions containing different endmembers. However, there are several situations where the LMM no longer functions well due to the breakdown of the used assumptions. One notable example are scenes with large geometrical structures such as buildings or trees, where shadowing and mutual illumination start to play a large role. Another example are mineral mixtures, where an incoming light ray can interact many times with the different mineral grains, and the single interactions assumed in the LMM can even become relatively rare [1]. Furthermore, the LMM only considers reflection and disregards optical transmission, which can become quite important in vegetation and mineral mixtures.

To cope with these additional complexities, more involved models have been developed over the years. These nonlinear models can be divided into several different categories, depending on the intended application or their complexity. In this work, we will focus mainly on the models used in remote sensing, and more specifically, hyperspectral image processing.

A first class of nonlinear models are the bilinear or linearquadratic models, which extend the LMM with additional terms that describe spectral interactions between two endmembers. Many such models have been introduced, each with its own mixing model, restrictions, and underlying physical reasoning. The model proposed by Nascimento [2] and Somers [3] assumes that the bilinear interaction terms can be considered as extra endmembers, leading to a LMM consisting of both linear and bilinear terms. Remark that this model has been proposed for two endmembers in [4] as well. Other approaches, such as the Fan model [5], the generalized bilinear model (GBM) [6] or the polynomial post-nonlinear model (PPNM) [7], [8] scale the bilinear interactions with the linear abundances, reasoning that the probability of interacting with two different materials should be proportional to their presence in the pixel. It is this class of bilinear models that we will consider in this paper, focusing especially on the PPNM model. Several other bilinear or linear-quadratic models can be found in [9], [10], and detailed overviews of these models and their differences can be found in the review papers [11], [12].

These bilinear models can in theory be extended to allow interactions between more than two endmembers by introducing trilinear or higher-order terms, or by using higher-order polynomials in the PPNM. However, it is argued in several of the papers on the bilinear models [4], [8] that such higher order terms do not contribute significantly to the spectral signal, as they involve multiplications of several reflectance 
and abundance values that are all smaller than one. Their contribution to the total spectral signal is not considered to be significant enough to merit their added complexity, as adding such higher order terms will usually lead to models that become very hard to solve. On the other hand, in certain other scenarios such as mineral mixtures involving very bright components [1], it is observed that multiple reflections will start to play a dominant role in the observed spectral signal, and third-order reflections or higher can no longer be simply dismissed. A similar reasoning can be made for vegetation canopies, where the often dense three-dimensional structures might be expected to generate a significant fraction of higherorder interactions.

Two broad and overlapping classes of nonlinear mixing models that take such higher-order interactions into account are, with increasing complexity, the layered models and the radiative transfer models. Both have been used to describe vegetation and mineral mixtures, and show sometimes dramatic improvements over linear mixing models. In layered models, one severely simplifies the geometrical description of a complex scene by modeling it as a stack of layers or plates. Depending on the model, techniques from radiative transfer, radiosity theory or transfer matrix techniques are used to derive the resulting mixture models. In all these layered models, transmission through layers is also taken into account. Some notable examples of mixing models based on a layered approach are the canopy model derived in [13] based on radiosity theory, the isograin model for mineral mixtures in [14], [15] which uses transfer matrices, or radiative transfer based models such as the Shkuratov model [16] and the model proposed in [17].

Many radiative transfer based models that do not assume a layered geometrical structure have been derived as well, and have reached very high levels of complexity, integrating a large amount of possible optical and physical effects that can be present in mineral mixtures, such as coherent backscattering, porosity, interference, the effects of grain sizes and shapes. One of the first such models which is also often used in hyperspectral unmixing was the Hapke model [1], which has been constantly refined since its inception. We do not aim to provide a detailed description of these types of models, but refer to the rich literature on these models. See e.g. [18], [19] and references therein. Furthermore, we refer to the overview paper [11] and references therein for a much more detailed explanation of many of the models and concepts described in this section.

One problem with layered or radiative transfer based models is that while they are excellent forward models, they can often not be easily used for hyperspectral unmixing. If all the physical properties of a scene and its components are known, these models excel at predicting the resulting reflectance. For the unmixing application however, we need to invert these models, as we want to know the abundances of the endmembers given only the observed reflectances. This is usually very hard to do without several strong additional assumptions, destroying much of the strength of such models.

In this work, we present a new model which takes multiple reflections into account: the multilinear mixing (MLM) model.
The MLM model can be considered as an extension of the bilinear models to include all orders of interactions, or as an extension of the PPNM to all degrees. The MLM model introduces but a single parameter which describes the probability of undergoing further interactions. This makes the model computationally and analytically tractable, and physically sound, as opposed to several other possible extensions of the bilinear models which are not physical or possess a high complexity. The model is not as advanced as several of the layered or radiative transfer based models, which is also one of its strengths: The resulting mixing equation can be easily inverted, and used for practical unmixing applications. Furthermore, the MLM model provides a per-pixel estimate of the size of the nonlinear component. This way, maps can be generated indicating where nonlinear effects manifest themselves, which can be useful information in many scenarios.

This paper is organized as follows: In section II we present several existing bilinear models, and show some difficulties one might encounter when these models are extended to an infinite number of interactions. In section III the MLM model is introduced. We first explain the underlying physical interpretation, show how a mixing model can be derived from these assumptions, and finally how this model can be inverted and solved given a data set and known endmembers. Next, several experiments on synthetic and real data follow in section IV, along with comparisons with several alternatives, such as the LMM, bilinear models, and the Hapke model for intimate mixtures. Section V contains the conclusions, followed by the bibliography.

\section{BILINEAR MODELS}

Consider a data matrix $X=\left(\boldsymbol{x}_{1}, \ldots, \boldsymbol{x}_{N}\right)$ containing columnwise the $N$ data points $\left\{\boldsymbol{x}_{i}\right\}_{i=1}^{N}$ in a $d$-dimensional spectral space, and $p$ endmembers listed columnwise in the endmember matrix $E=\left(\boldsymbol{e}_{1}, \ldots, \boldsymbol{e}_{p}\right)$. The LMM given in (1) then assumes that each data point can be constructed as a convex linear combination of endmembers, with their respective abundances as linear coefficients

$$
\boldsymbol{x}=\sum_{i=1}^{p} a_{i} \boldsymbol{e}_{i}+\boldsymbol{\eta}
$$

where also additive Gaussian noise is added to each band, represented by the noise vector $\boldsymbol{\eta}$. The constraints on the abundances are the ANC and ASC:

$$
\forall i: a_{i} \geq 0, \quad \sum_{i=1}^{p} a_{i}=1
$$

As noted in the introduction, this model cannot deal with multiple reflections that often occur in many situations, and several authors have hence augmented the LMM with bilinear terms. The Fan bilinear model [5] is given by

$$
\boldsymbol{x}=\sum_{i=1}^{p} a_{i} \boldsymbol{e}_{i}+\sum_{i=1}^{p-1} \sum_{j=i+1}^{p} a_{i} a_{j} \boldsymbol{e}_{i} \odot \boldsymbol{e}_{j}+\boldsymbol{\eta}
$$

where $\boldsymbol{x} \odot \boldsymbol{y}=\left(x_{1} y_{1}, \ldots, x_{d} y_{d}\right)$ is the Hadamard or element-wise product of two vectors. The constraints on the 
abundances are the traditional ANC and ASC also employed in the LMM (2). The bilinear terms in the Fan model are scaled with the respective abundances, reasoning that the probability that a light ray interacts with any two endmembers has to be proportional to their abundances in the scene.

The generalized bilinear model extends the Fan model by including additional free parameters $\gamma_{i j} \in[0,1]$ in each bilinear interaction:

$$
\boldsymbol{x}=\sum_{i=1}^{p} a_{i} \boldsymbol{e}_{i}+\sum_{i=1}^{p-1} \sum_{j=i+1}^{p} \gamma_{i j} a_{i} a_{j} \boldsymbol{e}_{i} \odot \boldsymbol{e}_{j}+\boldsymbol{\eta}
$$

These additional parameters allow more flexibility in the model, and allow for better spectral reconstructions. The amount of free parameters can become quite large for increasing values of $p$, which can cause numerical problems.

A linear-quadratic model that was recently proposed is the PPNM. The mixing model contains two steps: First, the spectrum is linearly mixed with the LMM. Next, a polynomial transformation of the resulting spectrum is performed to introduce nonlinearities. In [7], [8], only a second-order polynomial is assumed, leading to a linear-quadratic model, or a bilinear model which includes self-interactions. The modeled spectrum $\boldsymbol{x}$ is obtained as

$$
\begin{aligned}
\boldsymbol{x} & =\boldsymbol{y}+b(\boldsymbol{y} \odot \boldsymbol{y})+\boldsymbol{\eta} \\
\boldsymbol{y} & =\sum_{i=1}^{p} a_{i} \boldsymbol{e}_{i}
\end{aligned}
$$

with $b$ a parameter which scales the size of the quadratic term. By substitution, this mixing model can also be written as

$$
\boldsymbol{x}=\sum_{i=1}^{p} a_{i} \boldsymbol{e}_{i}+b \sum_{i=1}^{p} \sum_{j=1}^{p} a_{i} a_{j} \boldsymbol{e}_{i} \odot \boldsymbol{e}_{j}+\boldsymbol{\eta}
$$

Note that both the Fan model and the GBM exclude selfinteraction terms such as $\boldsymbol{e}_{i} \odot \boldsymbol{e}_{i}$, while these are included in the PPNM. It is noted in several papers [9], [20] that selfinteractions can form an important contribution in the total spectral signal, and should hence be allowed.

Furthermore, most images used in hyperspectral remote sensing are reflectance images, and the spectral vectors are restricted to the interval $[0,1]^{d}$. The mixing models should reflect this restriction, which is not the case in the GBM, PPNM or Fan model as these models can output modeled spectra outside this range.

Note that several other types of bilinear models exist as well, with sometimes very different interpretations or derivations [3], [2], [9], [10]. We will only consider bilinear models that scale the bilinear terms with the abundances.

Extending the models proposed in previous section with higher-order terms is not straightforward. Consider for instance the following noiseless mixing equation, which is the PPNM with $b=1$, or a version of the Fan model where selfinteractions are added:

$$
\boldsymbol{x}=\sum_{i=1}^{p} a_{i} \boldsymbol{e}_{i}+\sum_{i=1}^{p} \sum_{j=1}^{p} a_{i} a_{j} \boldsymbol{e}_{i} \odot \boldsymbol{e}_{j}
$$

If we extend this model to include higher order interactions, this becomes

$$
\begin{aligned}
\boldsymbol{x}= & \sum_{i=1}^{p} a_{i} \boldsymbol{e}_{i}+\sum_{i=1}^{p} \sum_{j=1}^{p} a_{i} a_{j} \boldsymbol{e}_{i} \odot \boldsymbol{e}_{j} \\
& +\sum_{i=1}^{p} \sum_{j=1}^{p} \sum_{k=1}^{p} a_{i} a_{j} a_{k} \boldsymbol{e}_{i} \odot \boldsymbol{e}_{j} \odot \boldsymbol{e}_{k}+\ldots
\end{aligned}
$$

or by using the same substitution (6) as in the PPNM

$$
\begin{aligned}
\boldsymbol{x} & =\boldsymbol{y}+(\boldsymbol{y} \odot \boldsymbol{y})+(\boldsymbol{y} \odot \boldsymbol{y} \odot \boldsymbol{y})+\ldots \\
& =\sum_{i=1}^{\infty} \boldsymbol{y}^{i}
\end{aligned}
$$

where all algebraic operations containing vectors have to be considered on a component-wise basis. As all components of $\boldsymbol{y}$ are in $[0,1]$, this leads to

$$
\begin{aligned}
\boldsymbol{x} & =\frac{\boldsymbol{y}}{1-\boldsymbol{y}} \\
& =\frac{\sum_{i=1}^{p} a_{i} \boldsymbol{e}_{i}}{1-\sum_{i=1}^{p} a_{i} \boldsymbol{e}_{i}}
\end{aligned}
$$

It is immediately clear that the resulting reflectance will not stay bounded to $[0,1]$, and that simply extending the Fan model to an infinite number of multilinear interactions will not give a realistic model.

\section{THE MULTILINEAR MIXING MODEL}

\section{A. Derivation of the model}

We propose a new nonlinear mixing model that solves several of the problems described in the previous section. The model includes all degrees of endmember interactions, and has a clear underlying physical reasoning. Furthermore, the reflectance vectors generated by the model stay constrained to the allowed interval $[0,1]$. This can be accomplished by considering the reflection process as a discrete Markov process.

We use a ray-based approximation of light, and trace the path that a single light ray follows before reaching the observer. This path is modeled as a discrete Markov chain, subject to the following rules:

- A light ray incoming from the source will interact with at least one material.

- After each interaction with a material, the ray will have a probability $P$ of undergoing further interactions, and a probability $(1-P)$ of escaping the scene and reaching the observer.

- The probability of interacting with material $i$ is proportional to its abundance $a_{i}$.

- When a light ray is scattered by material $i$, its intensity changes according to that material's albedo $\boldsymbol{w}_{i} \in[0,1]^{d}$. 


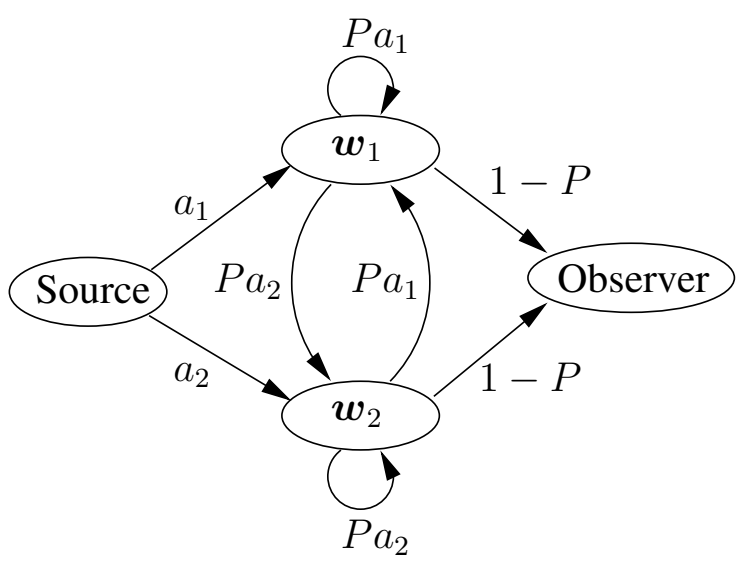

Fig. 1: Diagram describing the transition probabilities between source, endmembers and observer for a two-endmember scenario.

This process is illustrated for a two endmember scenario in the diagram in Fig. 1, where a directed graph is used to represent the transition probabilities. From this diagram, it can be easily seen that the probability that a light ray will follow a sequence $\left(\boldsymbol{w}_{i_{1}}, \boldsymbol{w}_{i_{2}}, \ldots, \boldsymbol{w}_{i_{R}}\right)$ before reaching the observer is given by

$\operatorname{Prob}\left(\boldsymbol{w}_{i_{1}}, \boldsymbol{w}_{i_{2}}, \ldots, \boldsymbol{w}_{i_{R}}\right)=(1-P) P^{R-1} a_{i_{1}} a_{i_{2}} \ldots a_{i_{R}}$

The spectral contribution of such a path is $\boldsymbol{w}_{i_{1}} \odot \boldsymbol{w}_{i_{2}} \odot \ldots \odot$ $\boldsymbol{w}_{i_{R}}$. The total observed reflectance under these assumptions then becomes the sum over all possible paths of these contributions, each weighted by their corresponding probability:

$$
\boldsymbol{x}=\sum_{R=1}^{\infty}\left(\sum_{i_{1}=1}^{p} \ldots \sum_{i_{R}=1}^{p}\right)(1-P) P^{R-1} \bigodot_{k=1}^{R}\left(a_{i_{k}} \boldsymbol{w}_{i_{k}}\right)
$$

This summation can be conveniently written as a multilinear expansion. The resulting MLM model becomes

$$
\begin{aligned}
\boldsymbol{x}= & (1-P) \sum_{i=1}^{p} a_{i} \boldsymbol{w}_{i}+(1-P) P \sum_{i=1}^{p} \sum_{j=1}^{p} a_{i} a_{j} \boldsymbol{w}_{i} \odot \boldsymbol{w}_{j} \\
& +(1-P) P^{2} \sum_{i=1}^{p} \sum_{j=1}^{p} \sum_{k=1}^{p} a_{i} a_{j} a_{k} \boldsymbol{w}_{i} \odot \boldsymbol{w}_{j} \odot \boldsymbol{w}_{k} \ldots \\
= & (1-P) \boldsymbol{y}+(1-P) P \boldsymbol{y}^{2}+(1-P) P^{2} \boldsymbol{y}^{3} \ldots \\
= & (1-P) \boldsymbol{y}+P \boldsymbol{y} \odot\left((1-P) \boldsymbol{y}+P(1-P) \boldsymbol{y}^{2} \ldots\right) \\
= & (1-P) \boldsymbol{y}+P \boldsymbol{y} \odot \boldsymbol{x}
\end{aligned}
$$

with $\boldsymbol{y}=\sum_{i} a_{i} \boldsymbol{w}_{i}$. The solution is given by

$$
\begin{aligned}
\boldsymbol{x} & =\frac{(1-P) \boldsymbol{y}}{1-P \boldsymbol{y}} \\
& =\frac{(1-P) \sum_{i=1}^{p} a_{i} \boldsymbol{w}_{i}}{1-P \sum_{i=1}^{p} a_{i} \boldsymbol{w}_{i}}
\end{aligned}
$$

Remark that in reality, spectral noise will be present, and a noise vector $\boldsymbol{\eta}$ has to be added to (15).

\section{B. Properties of the MLM model}

The reflectance values allowed by the mixing equation (15) of the MLM model will always lie in the interval $[0,1]$ for $P<1$. Remark that the model can be reduced to the LMM by choosing $P=0$. Furthermore, there is only a single parameter $P$ in addition to the set of abundances $\left\{a_{i}\right\}_{i=1}^{p}$, with a clear physical interpretation: $P$ is the probability of undergoing further interactions, and hence specifies the size of the nonlinear component in the model. This can be useful for detecting deviations from the LMM, and where nonlinear effects are present in an image. This possible use of the model will also be illustrated in the experiments.

The MLM model assumes that a light ray has a probability $P$ of undergoing further interactions at each step. The probability of reaching the observer at each interaction is a binomial trial with success rate $(1-P)$. The number of spectral interactions $X$ before reaching the observer follows a geometric distribution, as

$$
\text { Prob }(X=R)=(1-P) P^{R-1}
$$

The average number of interactions is given by

$$
E(X)=\frac{1}{1-P}
$$

It must be noted that the geometric distribution is a heavy tailed distribution, hence light rays undergoing many more interactions than this average can be expected.

The albedos $\left\{\boldsymbol{w}_{i}\right\}_{i=1}^{p}$ used in the model define how the light intensity changes when a ray bounces off the corresponding material, and play the role of endmembers in the model. Such endmembers are in practice often obtained from image pixels, as many endmember extraction algorithms (EEAs) employ the pure pixel assumption. Such a pure pixel might contain multiple reflections as well, and as such be a composite of single and multiple reflected paths. This distinction becomes important when the total contribution of multiply reflected paths becomes large, for instance in mineral mixture scenarios. The Hapke theory of reflectance also exploits this difference, and treats the albedo as a linear mixture instead of the observed total reflectance.

When only a single material with albedo $\boldsymbol{w}_{i}$ is present in a pixel, the mixing equation (15) reduces to

$$
\boldsymbol{e}_{i}=\frac{\left(1-P_{i}\right) \boldsymbol{w}_{i}}{1-P_{i} \boldsymbol{w}_{i}}
$$

which can be inverted as

$$
\boldsymbol{w}_{i}=\frac{\boldsymbol{e}_{i}}{P_{i} \boldsymbol{e}_{i}+1-P_{i}}
$$

It is thus in theory possible to determine the albedo $\boldsymbol{w}_{i}$ that corresponds with an observed endmember spectrum $\boldsymbol{e}_{i}$, as long as the variable $P_{i}$ is known beforehand. Furthermore, as each pure material might show a different reflectance-albedo relation, the $P_{i}$ values can be different for every endmember. 
This would introduce $p$ additional parameters in the mixing equation, one for each endmember, resulting in a much more involved model. Fortunately, in most practical situations in remote sensing, one can assume that the nonlinear effects are relatively small, which results in small values of $P_{i}$, and small differences between albedo and reflectance: $\forall i: \boldsymbol{e}_{i} \approx \boldsymbol{w}_{i}$. We will clearly indicate how we handled the reflectance-albedo relation in each of the experiments.

Furthermore, it must be noted that (15) is also well defined for $P<0$. Although the physical reasoning that led to (15) is no longer valid ( $P$ would become a negative probability), the mixing equation can in theory be used for negative values of $P$ as well, as the resulting reflectance values will still be restricted to the range $[0,1]$ for any $P<1$. The effect of the nonlinear behavior in this case would be to increase the total observed reflectance instead of causing a decrease with respect to the LMM result with the same endmembers and abundances. Such a situation might occur due to two reasons: A pixel might receive additional illumination from three-dimensional structures outside its IFOV, or the albedo vectors $\left\{\boldsymbol{w}_{i}\right\}_{i=1}^{p}$ are erroneous. The latter situation might occur if one or several materials do possess a high degree of multiple scattering, and a conversion from reflectance to albedo has not been performed.

An alternative motivation for relaxing the constraint on $P$ can be obtained by redefining the reflection process to include additional unknown scaling factors in each endmember spectrum, which will allow more flexibility in the model. Such an approach has been successfully used in the partially constrained least-squares unmixing algorithm [21] to deal with spectral variability, and in the Fan model [5] to obtain the GBM [6]. Remark that this is a reinterpretation of the reflection process which has no effect on the mixing equation (15), except for relaxing the constraint to $P<1$. Values for $P$ of one or larger are mathematically impossible as they will lead to singularities in (15).

An illustration of the data manifold generated by (15) is shown in Fig. 2, and the values of $\boldsymbol{x}$ as a function of $\boldsymbol{y}$ given by (14) are shown in Fig. 3. It can be seen from these figures that values of $P$ in $[0,1]$ will cause a decrease of the reflectance with respect to the LMM, while $P<0$ will cause an increase.

The abundances in the model now correspond with the probability that a light ray will interact with a given material. In the LMM, this corresponds with the fractional areal presence of the endmember in the pixel's field of view. In the MLM model this interpretation is not longer valid, and it is not sure that an abundance can be interpreted as a true areal fraction. Similar difficulties arise in other bilinear models [3]. Fortunately, the abundances in the MLM model still have a clear physical meaning, and also obey standard constraints such as the ANC and ASC. It does not seem unreasonable to assume a close similarity of the interaction probabilities in the MLM model with areal abundances, or other interpretations of abundances in spectral unmixing settings, such as volumetric fractions.

\section{Unmixing strategy}

To unmix a hyperspectral data set with the MLM model, we first assume that the albedos are available, e.g. obtained as

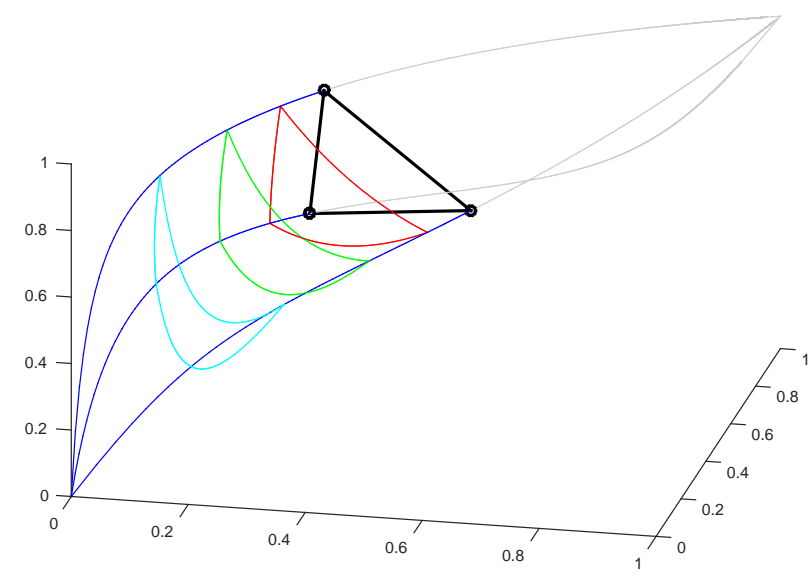

Fig. 2: Three endmembers in three dimensions (black circles), the paths that the endmembers follow when $P$ is varied in $[0,1]$ (blue) or for $P<0$ (gray), the manifold induced by the MLM model for $P=0$ (black), $P=0.25$ (red), $P=0.5$ (green) and $P=0.75$ (cyan).

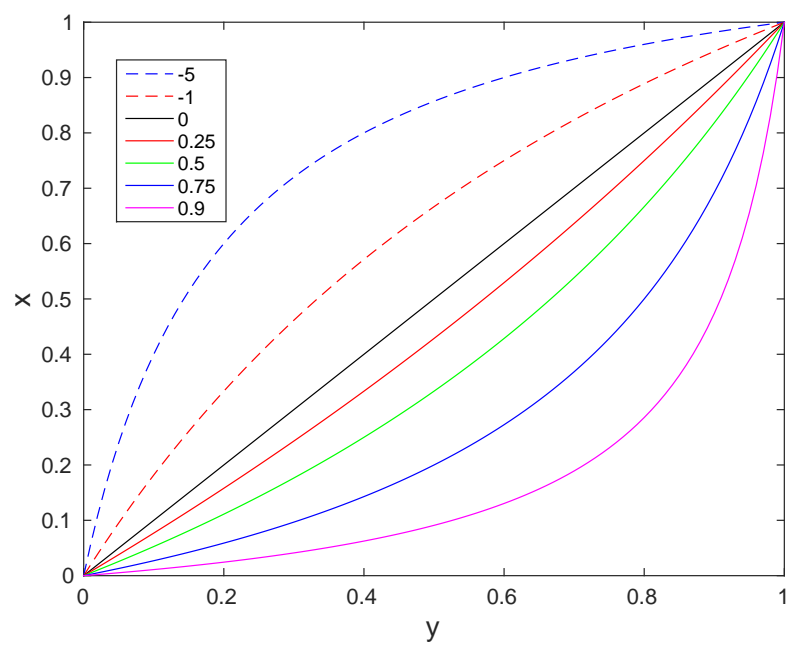

Fig. 3: The value of $x$ as a function of $y$ for different values of the parameter $P$, indicated in the legend.

endmember spectra with an EEA such as vertex component analysis (VCA) [22] or available from a library. Because we assume that the mixtures follow mixing equation (15), but are corrupted by additive uncorrelated Gaussian noise, we want to find the set of abundances $\left\{a_{i}\right\}_{i=1}^{p}$ and the value of $P$ that minimizes the reconstruction error (RE) with the data point $\boldsymbol{x}$. This is also the maximum likelihood solution under the employed mixing model and noise assumptions:

$$
\underset{\left\{a_{i}\right\}_{i=1}^{p}, P}{\operatorname{argmin}}\left\|\boldsymbol{x}-\frac{(1-P) \sum_{i=1}^{p} a_{i} \boldsymbol{w}_{i}}{1-P \sum_{i=1}^{p} a_{i} \boldsymbol{w}_{i}}\right\|_{2}^{2}
$$

For remote sensing imagery, we can set the albedos equal to the endmember spectra: $\forall i: \boldsymbol{w}_{i}=\boldsymbol{e}_{i}$, as we do not expect large values of $P$ in such data sets. On the other hand, in mineral mixtures the average number of spectral interactions can become very large, and if only endmember spectra are 


\begin{tabular}{|c|c|c|c|}
\hline Model & $\boldsymbol{x}$ & $\boldsymbol{\gamma}$ & Constraints \\
\hline LMM & $(1)$ & N/A & N/A \\
Fan & $(3)$ & N/A & N/A \\
GBM & $(4)$ & $\gamma=\left(\gamma_{i j}\right)_{i>j=1}^{p}$ & $\gamma_{i j} \in[0,1]$ \\
PPNM & $(7)$ & $b$ & N/A \\
MLM & $(15)$ & $P$ & $P \leq 1$ \\
\hline
\end{tabular}

TABLE I: The models, mixing equations, hyperparameters and constraints

available, a conversion to albedos should be carried out. An example of this situation is presented in section IV-C.

The abundances are subject to the ANC and ASC, while $P<1$. As the search space is a convex set, this minimization problem can be solved by constrained minimization techniques, such as active set approaches or quadratic programming.

Remark that this unmixing strategy can be generalized to other mixing models as well. Consider a data point $\boldsymbol{y}$ and a reconstructed point $\boldsymbol{x}(\boldsymbol{a}, \gamma)$ obtained with a mixing model which depends on the abundances in the vector $\boldsymbol{a}$ and on the hyperparameters in the vector $\gamma$ (and implicitly on the reflectances or albedos). The RE is then given by

$$
R(\boldsymbol{y}, \boldsymbol{a}, \boldsymbol{\gamma})=\|\boldsymbol{y}-\boldsymbol{x}(\boldsymbol{a}, \boldsymbol{\gamma})\|_{2}^{2}
$$

Remark that several authors do not square the norm in the right-hand side of (21), making RE and Euclidean distance equivalent. This does not affect the location of the minimum in parameter space. Next, for a given value of $\boldsymbol{y}$ and a given mixing model for $\boldsymbol{x}(\boldsymbol{a}, \boldsymbol{\gamma})$, we want to minimize $R(\boldsymbol{y}, \boldsymbol{a}, \boldsymbol{\gamma})$ with respect to $\boldsymbol{a}$ and $\gamma$. Depending on the mixing model used, several equality or inequality constraints are additionally present, which can be implemented via matrix identities or parameter ranges. The models used in the experimental section, with their mixing equation for $\boldsymbol{x}$, the hyperparameters $\gamma$, and the constraints, are given in table I. All models have the ANC and ASC on the abundance vector $\boldsymbol{a}$, given by (2).

In practice, we used the Matlab function FMINCON to perform the minimization of (21) with respect to $\boldsymbol{a}$ and $\gamma$, with the constraints on these variables implemented through the proper matrix identities. The employed technique is sequential quadratic programming, and the initial search point is given by $\boldsymbol{a}=p^{-1} \mathbf{1}$, and zero for every hyperparameter. The function tolerance was set to $10^{-10}$, while the constraint tolerance was set to $10^{-8}$. For all the results in all experiments in this paper, the minimizer found a local minimum within the tolerance limits. Remark that while the search space is always a convex set, the induced manifolds, and hence target functions, are not (see Fig. 2 for an example for the MLM model). Local minima of the objective function are possible in theory.

\section{EXPERIMENTS}

\section{A. Comparison with bilinear models on synthetic data}

We have tested the MLM model on several synthetic data sets, and compared the obtained results with some commonly used bilinear and linear-quadratic models. Three endmembers were randomly chosen from the USGS spectral database,

\begin{tabular}{|c|c|c|c|c|c|}
\hline & LMM & Fan & GBM & PPNM & MLM \\
\hline LMM & $0.00(0)$ & $0.030(15)$ & $0.00(0)$ & $0.00(0)$ & $0.00(0)$ \\
Fan & $0.10(10)$ & $0.00(0)$ & $0.00(0)$ & $0.00(0)$ & $0.023(13)$ \\
GBM & $0.026(43)$ & $0.009(9)$ & $0.00(0)$ & $0.00(0)$ & $0.006(7)$ \\
PPNM $^{1}$ & $0.12(11)$ & $0.004(8)$ & $0.02(7)$ & $0.00(0)$ & $0.025(17)$ \\
PPNM $^{2}$ & $0.038(4)$ & $0.086(28)$ & $0.038(4)$ & $0.00(0)$ & $0.013(4)$ \\
MLM & $0.15(47)$ & $0.19(47)$ & $0.15(47)$ & $0.05(22)$ & $0.00(0)$ \\
\hline
\end{tabular}

TABLE II: The mean RE for the noiseless data sets.

\begin{tabular}{|c|c|c|c|c|c|}
\hline & LMM & Fan & GBM & PPNM & MLM \\
\hline LMM & $0.00(0)$ & $0.18(11)$ & $0.00(0)$ & $0.00(0)$ & $0.00(0)$ \\
Fan & $0.17(10)$ & $0.00(0)$ & $0.00(0)$ & $0.022(16)$ & $0.14(8)$ \\
GBM & $0.10(7)$ & $0.087(70)$ & $0.00(0)$ & $0.014(11)$ & $0.073(54)$ \\
PPNM $^{1}$ & $0.16(9)$ & $0.040(35)$ & $0.029(41)$ & $0.00(0)$ & $0.13(8)$ \\
PPNM $^{2}$ & $0.24(13)$ & $0.35(21)$ & $0.24(13)$ & $0.00(0)$ & $0.13(9)$ \\
MLM & $0.18(18)$ & $0.30(19)$ & $0.18(18)$ & $0.10(13)$ & $0.00(0)$ \\
\hline
\end{tabular}

TABLE III: The mean AE for the noiseless data sets.

pruned to 50 wavelengths in the range 1.97-2.47 $\mu \mathrm{m}$, and 1000 abundance vectors were generated uniformly and randomly from the unit simplex, hence respecting the ANC and ASC. The data sets were generated using the mixing models themselves, i.e., the LMM, the Fan model, the GBM, the PPNM and the MLM model. The $\gamma$ parameters in the GBM were chosen randomly and uniformly in the interval $[0,1]$. Two different values were used for the $b$ parameter in the PPNM, $b=0.25$ and $b=-0.25$, indicated with PPNM ${ }^{1}$ and PPNM ${ }^{2}$ respectively. The $P$ values in the MLM data set were chosen randomly from a half-normal distribution with $\sigma=0.3$, and values of $P$ larger than one were set to zero. This creates mostly small values for $P$, which seems realistic for a real scenario. For all data sets, two variants were created: One without noise, and one with Gaussian noise with SNR $=20$.

These data sets were unmixed with respect to the known endmembers by optimization of the REs with respect to the abundances and possible model parameters. The reconstructions were calculated using the different mixing models, and possible model-dependent constraints were included in the optimization procedure. The performance of the unmixing procedure was assessed using the mean RE and the mean abundance error (AE). The mean RE is given by averaging (21) over all pixels. The $\mathrm{AE}$ is obtained as the absolute difference between the abundance values, averaged over all endmembers and data points. The obtained errors are listed in tables II and III for the noiseless data sets, and in tables IV and V for the noisy data sets, along with the uncertainty in the last digits as determined by the standard deviation. Each row corresponds with a data set, and each column with an unmixing model.

These results indicate that each model excels in unmixing its corresponding data sets. The GBM always outperforms the Fan model, which seems reasonable since the GBM extends

\begin{tabular}{|c|c|c|c|c|c|}
\hline & LMM & Fan & GBM & PPNM & MLM \\
\hline LMM & $0.009(2)$ & $0.038(17)$ & $0.009(2)$ & $0.009(2)$ & $0.009(2)$ \\
Fan & $0.11(10)$ & $0.011(3)$ & $0.011(3)$ & $0.011(3)$ & $0.033(16)$ \\
GBM & $0.033(40)$ & $0.020(10)$ & $0.009(3)$ & $0.010(3)$ & $0.016(8)$ \\
PPNM $^{1}$ & $0.13(12)$ & $0.016(11)$ & $0.035(80)$ & $0.011(3)$ & $0.037(21)$ \\
PPNM $^{2}$ & $0.045(6)$ & $0.093(30)$ & $0.045(7)$ & $0.006(2)$ & $0.020(6)$ \\
MLM & $0.16(46)$ & $0.20(46)$ & $0.16(46)$ & $0.06(21)$ & $0.007(3)$ \\
\hline
\end{tabular}

TABLE IV: The mean RE for the noisy data sets. 


\begin{tabular}{|c|c|c|c|c|c|}
\hline & LMM & Fan & GBM & PPNM & MLM \\
\hline LMM & $0.016(14)$ & $0.18(11)$ & $0.019(16)$ & $0.022(18)$ & $0.021(16)$ \\
Fan & $0.16(10)$ & $0.015(14)$ & $0.018(16)$ & $0.030(23)$ & $0.14(9)$ \\
GBM & $0.097(72)$ & $0.090(72)$ & $0.021(17)$ & $0.024(19)$ & $0.071(57)$ \\
PPNM $^{1}$ & $0.16(9)$ & $0.044(40)$ & $0.041(46)$ & $0.019(15)$ & $0.13(8)$ \\
PPNM $^{2}$ & $0.24(13)$ & $0.35(21)$ & $0.24(12)$ & $0.025(20)$ & $0.14(9)$ \\
MLM & $0.18(19)$ & $0.30(20)$ & $0.18(19)$ & $0.11(13)$ & $0.018(14)$ \\
\hline
\end{tabular}

TABLE V: The mean AE for the noisy data sets.

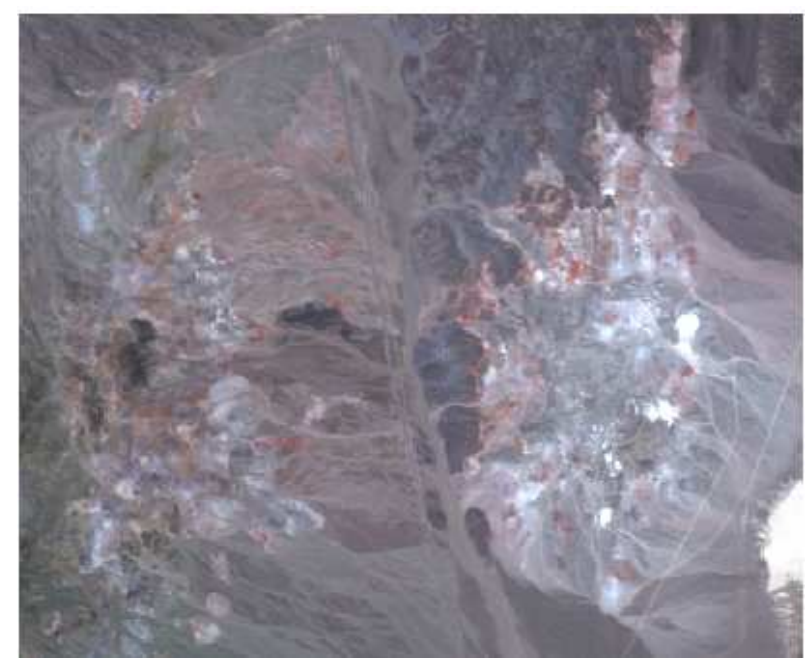

Fig. 4: The AVIRIS Cuprite data set in approximate true colors.

the Fan model with extra degrees of freedom. The PPNM performs significantly better than the bilinear alternatives on many data sets.

The MLM model outperforms the LMM and the Fan model on most data sets, and also the GBM on the PPNM data set with negative $b$. None of the bilinear models cope well on the MLM data set. As several of the considered bilinear models (Fan, GBM and PPNM with $b>0$ ) regard the bilinear interactions as a purely additional signal on top of the linear component, the inclusion of bilinear interactions will only allow reflectance values larger than those obtained via the LMM. On the other hand, the proposed MLM model will obtain smaller reflectance values for $P>0$ when compared to the LMM. These observations indicate that we need to allow negative values for $P$ in the MLM model for unmixing artificial data sets mixed according to several of these bilinear mixing models. One notable exception is the PPNM with negative values of $b$. Here, the total reflectance will decrease with respect to the LMM, and the MLM model will also obtain better reconstructions and abundance estimates than the bilinear alternatives.

\section{B. Real hyperspectral imagery}

1) The Cuprite data set: We have unmixed the well-known Cuprite data set obtained by the Airborne Visible/Infrared Imaging Spectrometer (AVIRIS), and displayed in Fig. 4. This data set has an IFOV of $20 \times 20 \mathrm{~m}^{2}$ per pixel, and images an area of the Nevada desert, USA, with many interesting geological features. The number of spectral bands is $d=51$, in the wavelength range $1.97-2.48 \mu \mathrm{m}$.
We unmixed this data set with the MLM model, and have used the several alternatives from the bilinear and linearquadratic family for comparison. First of all, 10 endmembers were extracted by the VCA algorithm [22], and identified by locating the spectra of minimal spectral angle in the USGS spectral database. Next, the abundance maps were generated using the bilinear mixing models used in previous subsection. Additionally, we also unmixed this data set with the Hapke unmixing methodology used in many papers [1], [23], [24], [11]. The Hapke model assumes that a mixture with a wavelength-dependent albedo $w$ will give rise to an observed reflectance $x$ through the relation

$$
x=\frac{w}{(1+2 \mu \sqrt{1-w})\left(1+2 \mu_{0} \sqrt{1-w}\right)}
$$

where $\mu$ and $\mu_{0}$ are the cosines of the angles of the incoming and outgoing light ray with the surface normal. These angles can be obtained from the metadata of the Cuprite data set, where we assume that the scene is flat. Linear constrained unmixing is applied to the albedo vectors instead of the reflectances, where (22) and its inverse are used for the conversion. A detailed explanation of this method of unmixing can be found in [25] and [11].

The abundance maps for four well-known minerals are presented in Fig. 5 for all mixing models. Unfortunately, no ground truth exists for the Cuprite data set, hence we cannot perform a quantitative analysis of these abundance maps. However, it should be clear that the abundance maps obtained by the different methods show subtle differences. The Hapke model shows more speckle noise than the LMM, and the mizzonite endmember has a much lower overall abundance. Also the PPNM and the Fan model show significant differences in the mizzonite abundance map. The MLM model abundance maps show a large overlap with the LMM maps, but also some significant differences in small areas of the image.

As indicated in section III, the $P$ values obtained by the MLM model have a clear physical meaning, at least for the interval $[0,1]$. They indicate the probability that a light ray will undergo further reflections after each interaction, and can thus be used to assess the size of the effects of nonlinearity in a pixel, or the size of the deviation from the LMM. A similar approach was presented in [26], where a nonlinearity detector was developed based on the $b$ values found by the PPNM: Above a given threshold for $|b|$, the pixel is considered nonlinearly mixed.

We can compare the two approaches for nonlinearity detection by studying the $P$ values obtained by the MLM model, and the $b$ values obtained by the PPNM model. Proper thresholding is required to obtain an actual detector, and we refer to [26] for the underlying theory and practical implementation of determining an appropriate threshold.

In Fig. 6, we have plotted the histograms of the $P$ and $b$ values obtained in the Cuprite data set. It can be seen that $33 \%$ of the pixels has $P<0$, and that the $P$ values generally stay small. This is physically plausible, as one would not typically expect a very large fraction of light rays to undergo multiple interactions. For instance, ray tracing results present in the 

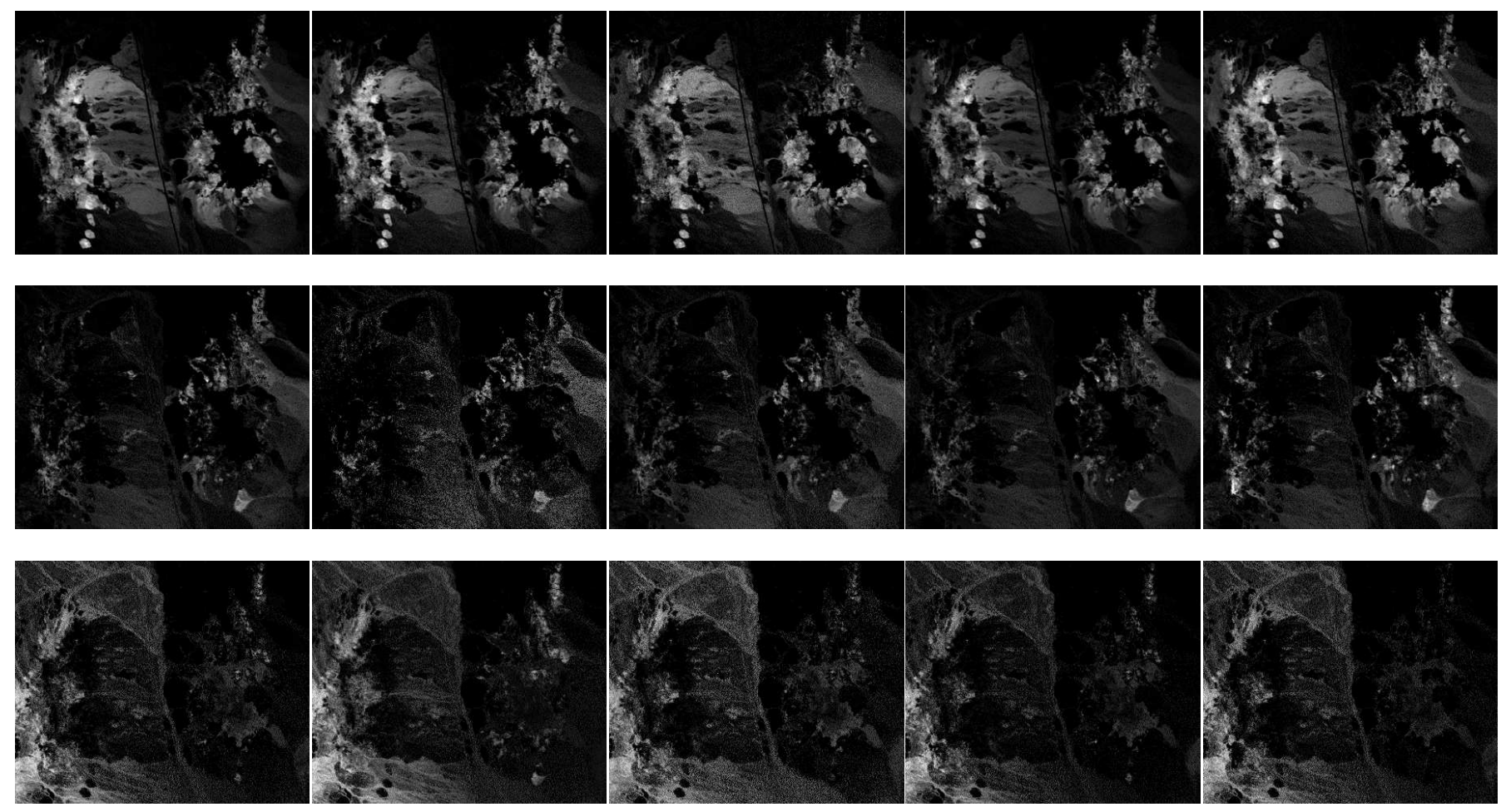

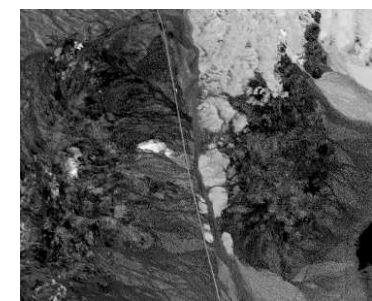

LMM

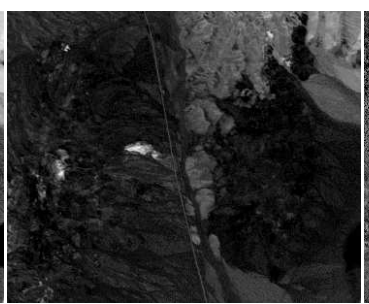

Hapke

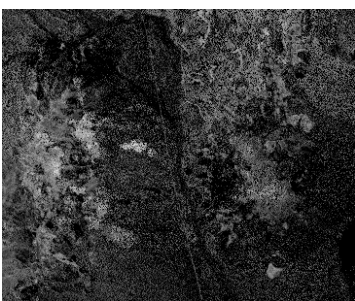

PPNM

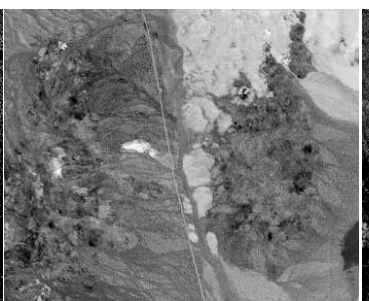

FM

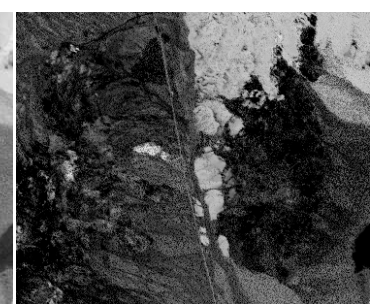

MLM

Fig. 5: The abundance maps of (top to bottom) alunite, kaolinite, hydrogrossular and mizzonite in the Cuprite data set, obtained by the (left to right) LMM, Hapke model, PPNM, FM and the MLM model.
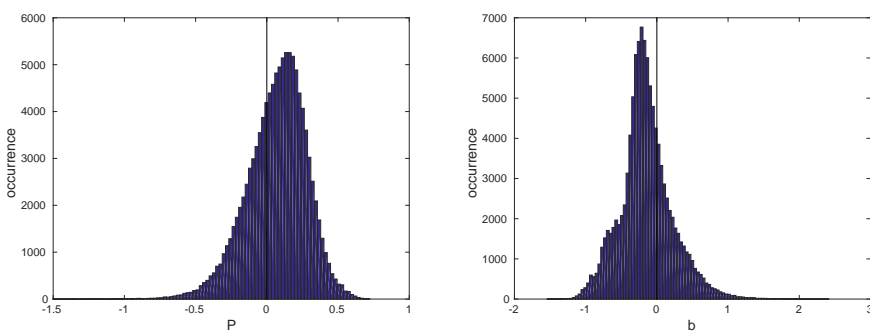

Fig. 6: Histogram of the values of $P$ obtained by the MLM model (left) and the values of $b$ obtained by the PPNM (right) on the Cuprite data set.

literature [27] show that a fraction of up to $20 \%$ of light rays will undergo multiple interactions in a vegetation scenario. The histogram for $b$ shows a similar situation, as most of the $b$ values also stay small.

These values can also be plotted for each pixel as color maps (Fig. 7), so that one can observe where the nonlinear effects will be the strongest. The map for the $P$ values obtained by the MLM model shows several contiguous areas where relatively strong nonlinear effects seem to be present. These areas are the areas where the abundance maps show the largest differences (see the alunite and kaolinite abundance maps in Fig. 5). Also the asphalt road crossing north-south, and the dirt road in the north, seem to show strong nonlinear effects. For the PPNM model, the results are less clear, as the negative $b$ values seem to correspond well with the mizzonite abundance map (see Fig. 5). While some overlaps can be observed between the two methods, no strong conclusions can be drawn from these results.

2) Heathland data set: The proposed unmixing methodology, along with nonlinearity detection, has been applied to the "Kalmthoutse heide" data set, which is a hyperspectral image of a heathland area in the north of Belgium, containing 52 bands in the wavelength range $0.45-2.55 \mu \mathrm{m}$, and a IFOV of approximately $2.4 \times 2.4 \mathrm{~m}^{2}$. We focus on a subsection of this image of $200 \times 200$ pixels, displayed in Fig. 8 (a). This part of the image contains standalone trees with visible shadows, 

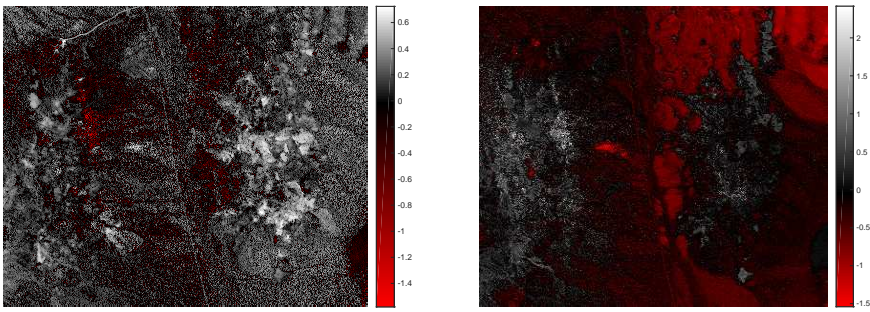

Fig. 7: The values of $P$ obtained by the MLM model (left) and $b$ obtained by the PPNM (right) for each pixel. Positive (negative) values are indicated as gray (red) values.

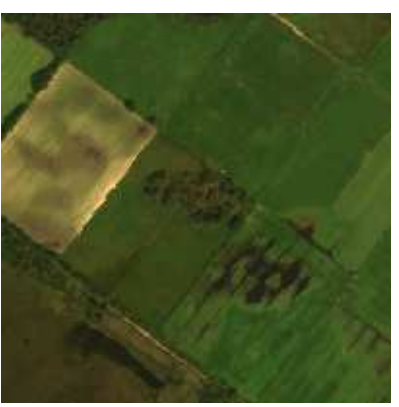

(a)

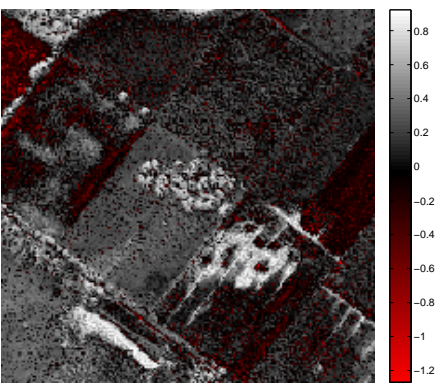

(b)
Fig. 8: (a): The heathland data set in approximate true colors. (b): The values of $P$ obtained by the MLM model.

forest, water bodies, and partly submerged grasslands, all of which can be expected to generate optically complex nonlinear effects to some extent.

We extracted 8 endmembers from this data set with the VCA algorithm. Detailed and hierarchical classification ground truth exists for this data set [28], with 24 classes on the finest classification level, describing different types of vegetation, grasslands, heathlands, arable fields, forests, and water bodies. This classification ground truth was used to identify the obtained endmembers. Three pairs of endmembers were identified with the same class, which lead to five uniquely identified classes: Temporary grassland, dry natural permanent grassland, Juncus effusus-dominated grassland (common rush), shallow water, and arable field with maize. The abundance maps of these 5 classes are displayed in Fig. 9, where we summed the abundance maps of classes with two endmembers together.

These abundance maps can be visually compared with Fig. 8 (a), and show some clear differences in certain areas. Especially around the water bodies, large differences can be seen: The MLM model is able to find strips of common rush, a vegetation commonly found on moist soil, around and in these water bodies. The LMM detects the trees in the image as water, while the MLM model does not show this behavior (note that a tree or forest endmember was not obtained by the VCA endmember extraction). Also the dry grassland is much more prominently present in the MLM abundance maps.

The $P$ values obtained by the MLM model are displayed in Fig. 8 (b), and can be used to assess the size of the nonlinear effects in the image. This map corresponds very well with the areas in the image where large nonlinear effects can be

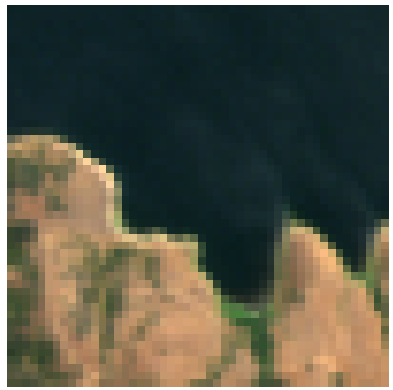

(a)

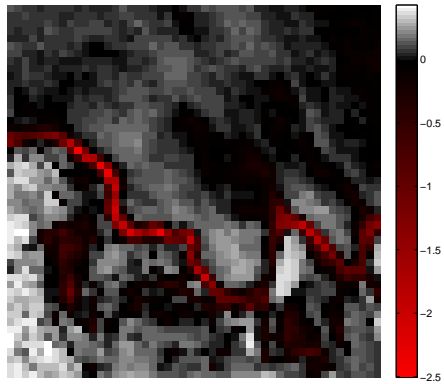

(b)
Fig. 10: (a): The Moffett Field data set in approximate true colors. (b): The values of $P$ obtained by the MLM model.
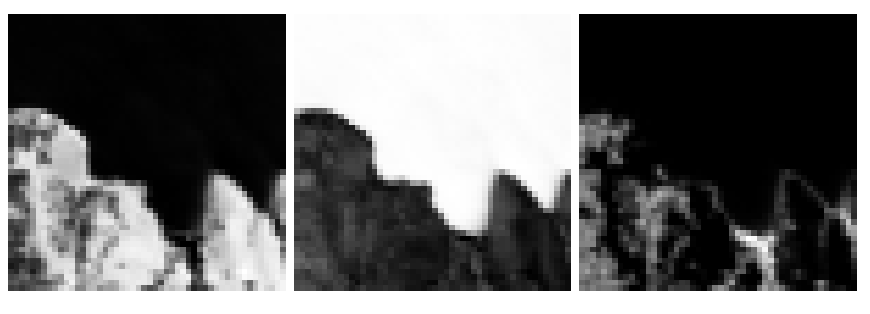

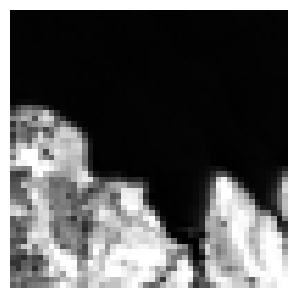

Soil

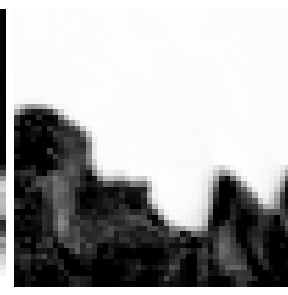

Water/shadow

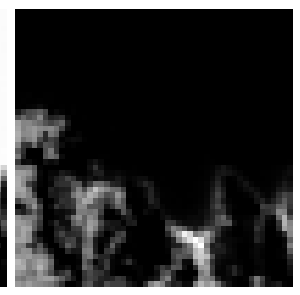

Vegetation
Fig. 11: The three abundance maps of the Moffett Field data set. Top: the LMM. Bottom: The MLM model.

expected, such as in the shallow water bodies, and in the trees, forest and their shadows. These are also the areas where the abundance maps show the largest differences.

3) Moffett field data set: Another data set which is used by many authors to assess spectral unmixing techniques [29], [30], [31] is the AVIRIS Moffett Field data set, displayed in Fig. 10 (a). We focus on a $50 \times 50$ pixel subsection of this image, as this subsection has also been used to assess the performance of several bilinear models [6], [32].

Three endmembers were extracted from this data set with the VCA algorithm. Comparison of their spectra and abundance maps with results in the literature [6], [32] show that these endmembers correspond to soil, water and vegetation. With these endmembers, we have unmixed the data set with the LMM and the MLM model. The abundance maps are shown in Fig. 11, and the map for the $P$ values is shown in Fig. 10 (b).

While the abundance maps show but subtle differences, the map for $P$ clearly indicates the areas where nonlinear effects can be expected. Note that the coastline shows relatively large negative values for $P$, indicating that the observed spectra here are larger in magnitude than any convex linear combination of 

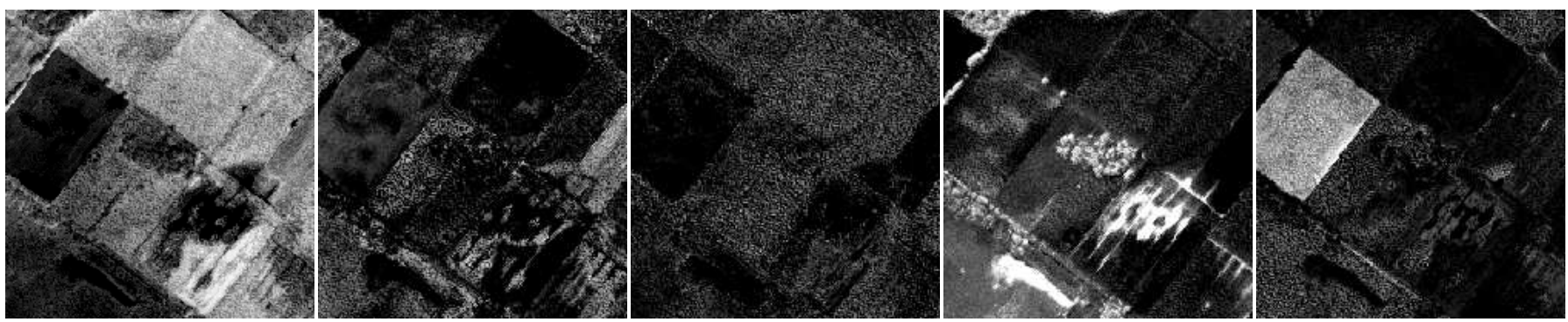

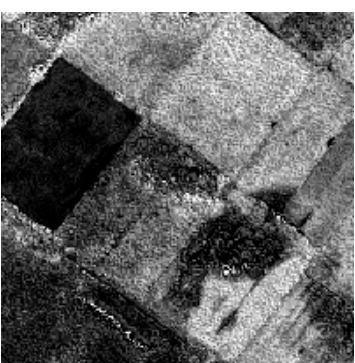

Temporary grassland

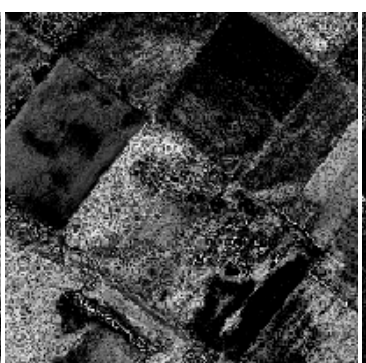

Dry grassland

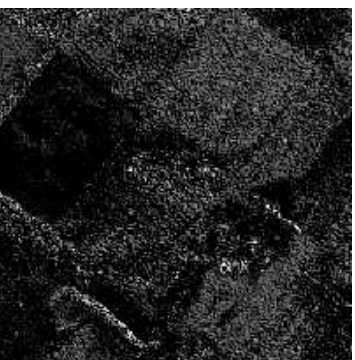

Common rush

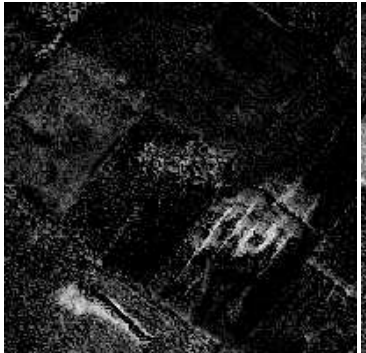

Shallow water

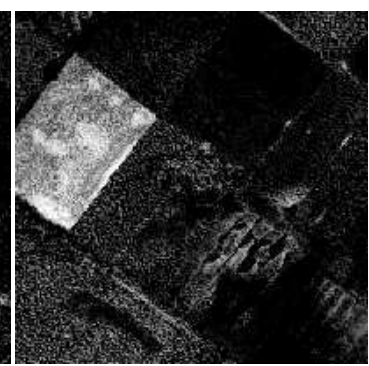

Arable maize

Fig. 9: The abundance maps of the 5 classes. Top: the LMM. Bottom: The MLM model.

endmember spectra. Furthermore, as the spectrum of the water endmember has a very small reflectance for all wavelengths, it can also be interpreted as a shadow endmember.

\section{Intimate mineral mixtures}

The performance of the MLM model can be tested on intimate mineral mixtures measured in a laboratory setting as well. One drawback is that the unmixing strategy that was employed for hyperspectral imagery will fail to provide better results than the LMM on such data sets, because one can no longer assume that the albedos used in the MLM model are equal to the measured reflectances of the pure mineral components. By using alternative theories for the optical effects in mineral mixtures, such as diffusive reflectance or Hapke's isotropic multiple scattering approximation, one can estimate several of the parameters we would need in the MLM model.

In [33], several relations between (single scattering) albedo and reflectance are provided. For instance, by using diffusive reflectance, one can derive that an observed reflectance of 0.8 in an intimate mineral mixture corresponds with an albedo of 0.99 , and 18 reflections on average before reaching the observer. A series distribution of the reflectance-albedo relation (22) derived by Hapke shows that the albedo is nine times larger than reflectance to first order (valid for low reflectance values), and that higher order terms have a large contribution for higher albedos, indicating the importance of multiply reflected paths. All these results suggest that values of $P$ close to one would be required for intimate mixtures.

In Fig. 12, we have plotted the relation between reflectance and albedo given by the Hapke model. We have also added this relation as given by the MLM equation (15). Notice that these plots follow the same trend, but that large values for $P$

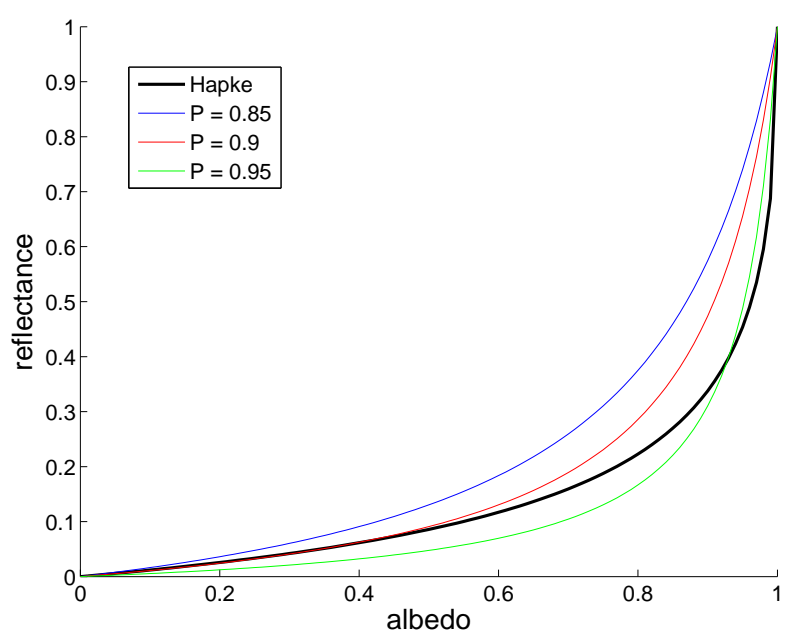

Fig. 12: The reflectance-albedo relation from the Hapke model (with $\mu=\mu_{0}=1$ ), and the MLM model for several large values of $P$.

are required, indeed indicating that multiple reflections play a dominant role in intimate mixtures.

For unmixing mineral mixtures with the MLM model, one can follow the same strategy as employed in the Hapke model. All reflectances are converted to albedo using a reflectancealbedo relation, the albedos are linearly unmixed and reconstructed using the LMM, and the reconstructed albedo is converted to reflectance again. If this strategy is used with the Hapke model, relation (22) is used to convert between reflectances and albedos. If we employ the MLM model, equation (14) is used. However, the MLM model depends on the $P$ variable, which is unknown beforehand. We assume that the value of $P$ which yields the smallest $\mathrm{RE}$ is the most plausible, and optimize with respect to the abundances and $P$. Remark 


\begin{tabular}{|c|c|c|c|c|c|}
\hline True & 1 & 0.75 & 0.5 & 0.25 & 0 \\
\hline LMM & 1.00 & 0.34 & 0.21 & 0.075 & 0 \\
GBM & 1.00 & 0.32 & 0.17 & 0.071 & 0 \\
PPNM & 1.00 & 0.32 & 0.18 & 0.073 & 0 \\
Hapke & 1.00 & 0.73 & 0.51 & 0.26 & 0 \\
MLM & 1.00 & 0.49 & 0.39 & 0.12 & 0 \\
$P$ value & 0 & 0.71 & 0.88 & 0.60 & 0 \\
\hline
\end{tabular}

TABLE VI: The abundance of the quartz component in a quartz-alunite binary mixture. The optimal $P$ values are given as well.

that the same value for $P$ is used for both the endmembers $\left\{\boldsymbol{e}_{i}\right\}_{i=1}^{p}$ and the target spectrum $\boldsymbol{x}$. This will simplify the optimization and avoids overfitting. Furthermore, because all mineral components in the employed binary mixture share similar physical properties [14], also their nonlinear behavior can be expected to be similar. The optimization problem then becomes

$$
\underset{\left\{a_{i}\right\}_{i=1}^{p}, P}{\operatorname{argmin}}\left\|\boldsymbol{x}-\frac{(1-P) \sum_{i=1}^{p} \frac{a_{i} \boldsymbol{e}_{i}}{P \boldsymbol{e}_{i}+1-P}}{1-P \sum_{i=1}^{p} \frac{a_{i} \boldsymbol{e}_{i}}{P \boldsymbol{e}_{i}+1-P}}\right\|
$$

To demonstrate this unmixing strategy, we have unmixed quartz-alunite mineral mixtures from the RELAB spectral database. These mixtures are described in detail in [14], and show highly nonlinear mixing behavior due to the presence of the quartz component, which has a very high albedo. As these mixtures were carefully crafted in a laboratory setting, the true abundances are known.

The abundances obtained by different unmixing methods are shown in table VI, along with the optimal values for $P$ obtained by the MLM model. These results indicate that the LMM, the GBM and the PPNM all show severe deviations from the true abundances. The Hapke model shows excellent performance on this data set, and obtains abundances which are almost indiscernible from the ground truth. The MLM model also shows a systematic deviation from the ground truth, but performs much better than the linear of bilinear alternatives. As this data set is a prime example of an intimate mineral mixture, it can be expected that models designed for unmixing remotely sensed imagery will not perform well. The MLM model somewhat mitigates this because it is able to interpolate between linear mixing and mixing including multiple reflections by modifying its $P$ parameter. This is clear from the improved results, but the performance of much more involved models specifically designed for intimate mixtures, such as the Hapke model, cannot be reached.

\section{Conclusions}

In this work, we have derived a new nonlinear mixing model which extends the existing bilinear mixing models to an infinite number of reflections. The model is based on a Markov chain interpretation of the reflection process of a single light ray, and introduces an additional parameter which describes the probability of undergoing further interactions after a reflection. This interpretation leads to a mixing equation which can be inverted via a constrained optimization technique. Several problems which might pose themselves in bilinear models are not present in this model, as every parameter has a clear physical interpretation, and the reflectances produced by the mixing equation stay constrained to the allowed interval $[0,1]$.

The model is demonstrated on several synthetic data sets, on three hyperspectral images, and on spectra of intimate mineral mixtures. Furthermore, plotting the value of the nonlinearity parameter for each pixel in a hyperspectral image results in maps that indicate where nonlinear behavior is present in the scene.

Future work concerns further theoretical and practical comparison of the model with more advanced models for vegetation or mineral mixing, such as models based on radiative transfer or layered approaches. In many situations, transmittance plays a large role, and transmission effects could be incorporated into the model. Finally, wavelength- or albedodependence of the nonlinear optical effects might be present, and can be investigated with such models as well.

\section{ACKNOWLEDGMENTS}

We would like to thank the anonymous reviewers for interesting suggestions for improvement of the manuscript, and the Flemish institute for technological research (VITO) for use of the "Kalmthoutse heide" data set. Rob Heylen is a postdoctoral researcher sponsored by the Flemish fund for scientific research (FWO - Vlaanderen).

\section{REFERENCES}

[1] B. Hapke, "Bidirectional reflectance spectroscopy. 1. Theory," $J$ Geophys. Res., vol. 86, pp. 3039-3054, 1981.

[2] J.M.P. Nascimento and J.M. Bioucas-Dias, "Nonlinear mixture model for hyperspectral unmixing," Image Sig. Process. for Remote Sens. XV, vol. 7477, no. 1, pp. 74770I, 2009.

[3] B. Somers, K. Cools, S. Delalieux, J. Stuckens, D. Van der Zande, W.W. Verstraeten, and P. Coppin, "Nonlinear hyperspectral image analysis for tree cover estimates in orchards," Remote Sens. Environ., vol. 113, pp. 1183-1193, 2009.

[4] R.B. Singer and T.B. McCord, "Mars: Large scale mixing of bright and dark surface materials and implications for analysis of spectral reflectance," Proc. 10th Lunar Planetary Sci. Conf., pp. 1835-1848, 1979.

[5] W. Fan, B. Hu, J. Miller, and M. Li, "Comparative study between a new nonlinear model and common linear model for analysing laboratory simulated forest hyperspectral data," Int. J. remote sens., vol. 30, no. 11, pp. 2951-2962, 2009.

[6] A. Halimi, Y. Altmann, N. Dobigeon, and J.-Y. Tourneret, "Nonlinear unmixing of hyperspectral images using a generalized bilinear model," IEEE Trans. Geosci. Remote Sens., vol. 49, no. 11, pp. 4153-4162, 2011.

[7] Y. Altmann, A. Halimi, N. Dobigeon, and J.-Y. Tourneret, "Supervised nonlinear spectral unmixing using a polynomial post nonlinear model for hyperspectral imagery," IEEE proc. ICASSP, pp. 1009-1012, may 2011.

[8] Y. Altmann, A. Halimi, N. Dobigeon, and J.-Y. Tourneret, "Supervised nonlinear spectral unmixing using a post-nonlinear mixing model for hyperspectral imagery," IEEE Trans. Image Process., vol. 21, no. 6, pp. 3017-3025, Jun 2012.

[9] X. Chen and L. Vierling, "Spectral mixture analysis of hyperspectral data acquired using a tethered balloon," Remote Sens. Environ., vol. 103, pp. 338-350, 2006.

[10] I. Meganem, P. Déliot, X. Briottet, Y. Deville, and S. Hosseini, "Linearquadratic mixing model for reflectances in urban environments," IEEE trans. geosci. remote sens., vol. PP, no. 99, pp. 1-15, 2013.

[11] R. Heylen, M. Parente, and P. Gader, "A review of nonlinear hyperspectral unmixing methods," IEEE J. Sel. Top. Appl. Earth Obs. Remote Sens., vol. 7, no. 6, pp. 1844-1868, June 2014.

[12] N. Dobigeon, J.-Y. Tourneret, C. Richard, J.C.M. Bermudez, S. McLaughlin, and AO. Hero, "Nonlinear unmixing of hyperspectral images: Models and algorithms," IEEE Sig. Process. Mag., vol. 31, no. 1, pp. 82-94, Jan 2014. 
[13] C.C. Borel and S.A.W. Gerstl, "Nonlinear spectral mixing models for vegetative and soil surfaces.," Remote Sens. Environ., vol. 47, no. 3, pp. 403-416, 1994.

[14] T. Hiroi and C.M. Pieters, "Effects of grain size and shape in modeling reflectance spectra of mineral mixtures," Proc. Lunar Planetary Sci., vol. 22, pp. 313-325, 1992.

[15] M. Kinoshita and M. Miyamoto, "A model for analysis of the spectral reflectance of mineral mixtures," Proc. Symp. Antarctic Meteorites, vol. 3, pp. 230-239, 1990.

[16] Y.G. Shkuratov, L. Starukhina, H. Hoffmann, and G. Arnold, "A model of spectral albedo of particulate surfaces: Implications for optical properties of the moon," Icarus, vol. 137, pp. 235-246, 1999.

[17] S. Douté and B. Schmitt, "A multilayer bidirectional reflectance model for the analysis of planetary surface hyperspectral images at visible and nearinfrared wavelengths,' J. Geophys. Res., vol. 103, pp. 31367-31390, 1998.

[18] M.K. Shepard and P. Helfenstein, "A laboratory study of the bidirectional reflectance from particulate samples," Icarus, vol. 215, no. 2, pp. 526$533,2011$.

[19] M.K. Shepard and P. Helfenstein, "A test of the Hapke photometric model," J. Geophys. Res., vol. 112, no. E03001, pp. 17, 2007.

[20] N. Raksuntorn and Q. Du, "Nonlinear spectral mixture analysis for hyperspectral imagery in an unknown environment," IEEE Geosci. Remote Sens. Lett., vol. 7, no. 99, pp. 836-840, 2010.

[21] M.A. Veganzones, L. Drumetz, G. Tochon, M. Dalla Mura, A. Plaza, J.M. Bioucas-Dias, and J. Chanussot, "A new extended linear mixing model to address spectral variability," Proc. IEEE WHISPERS, jun 2014

[22] J.M.P. Nascimento and J.M. Bioucas-Dias, "Vertex component analysis: a fast algorithm to unmix hyperspectral data," IEEE Trans. Geosci. Remote Sens., vol. 43, no. 4, pp. 898-910, 2005.

[23] J.F. Mustard and C.M. Pieters, "Photometric phase functions of common geologic minerals and applications to quantitative analysis of mineral mixture reflectance spectra," J. Geophys. Res.: Solid Earth, vol. 94, no. B10, pp. 13619-13634, 1989.

[24] J.M.P. Nascimento and J.M. Bioucas-Dias, "Unmixing hyperspectral intimate mixtures," Proc. SPIE, Image and Signal Processing for Remote Sensing XVI, vol. 7830, pp. 78300C, 2010.

[25] J.F. Mustard and C.M. Pieters, "Quantitative abundance estimates from bidirectional reflectance measurements," J. Geophys. Res.: Solid Earth, vol. 92, no. B4, pp. E617-E626, 1987.

[26] Y. Altmann, N. Dobigeon, and J.-Y. Tourneret, "Nonlinearity detection in hyperspectral images using a polynomial post-nonlinear mixing model," IEEE Trans. Image Process., vol. 22, no. 4, pp. 1267-1276, 2013.

[27] P.V. Villeneuve, S.A. Gerstl, and G.P. Asner, "Estimating nonlinear mixing effects for arid vegetation scenes with MISR channels and observation directions," Proc. IEEE IGARSS, vol. 3, pp. 1234-1236, 1998.

[28] G. Thoonen, T. Spanhove, J. Vanden Borre, and P. Scheunders, "Classification of heathland vegetation in a hierarchical contextual framework," Int. J. Remote Sens., vol. 34, pp. 96 - 111, 2013.

[29] Suresh Subramanian, Nahum Gat, Michael Sheffield, Jacob Barhen, and Nikzad Toomarian, "Methodology for hyperspectral image classification using novel neural network," Proc. SPIE, vol. 3071, pp. 128-137, 1997.

[30] N. Dobigeon, S. Moussaoui, M. Coulon, J.-Y. Tourneret, and A. O. Hero, "Joint Bayesian endmember extraction and linear unmixing for hyperspectral imagery," IEEE Tran Sig. Proc., vol. 57, no. 11, pp. 43554368, 2009.

[31] O. Eches, N. Dobigeon, and J.-Y. Tourneret, "Enhancing hyperspectral image unmixing with spatial correlations," IEEE Trans. Geosci. Remote Sens., vol. 49, no. 11, pp. 4239-4247, Nov 2011.

[32] Y. Altmann, N. Dobigeon, and J.-Y. Tourneret, "Bilinear models for nonlinear unmixing of hyperspectral images," Proc. IEEE Whispers, pp. 1-4, 2011.

[33] B. Hapke, Theory of Reflectance and Emittance Spectroscopy, Top. Remote Sens. Cambridge University Press, 2005. 